objective of workforce planning is to develop knowledge and intelligence data on the workforce, to inform decisions at local level and to drive improvements in Occupational Health service outcomes.

Methods The Workplace Health and Wellbeing Unit was set up in March 2016 under Priority 2 Staff Engagement within the Health Services People Strategy 2015-2018. [www.hse.ie].

With reference to the HSE People Strategy 2015-2018 'We have clearly defined workforce planning as a key component of our people'

Occupational Health Services is one of four areas of governance and responsibility.

A mapping exercise of current Occupational Health Physician establishments and a profiling of activity in the occupational health departments were conducted.

A literature review of best practice in models of care was completed.

An agreed framework for workforce planning was adhered to.

Result A position paper on The Future Requirements for Occupational Health Physicians in the Health Service, Ireland has been submitted to relevant decision makers.

Regular review of the progression of this work will take place.

Discussion Occupational Health Services are reasonably well established in the Irish health care setting, however the future of the services have never been defined using a planned approach at national level As such this is the first initiative to identify and calculate the requirements for the future that ensure a sustainable future for the specialty and the service.

The position paper presented information on current Occupational Health Physician Staffing Establishments in the Irish Health Service, and it proposes a model of care for future delivery of Occupational Health Services provision in the Health Service in Ireland, and finally it articulates WHWU'S approach to maximise Occupational Health Physician roles in the context of workforce planning within the service.

\section{CLINICAL DIAGNOSTICS OF WORKING WOMEN EXPOSED TO ORGANOPHOSPHATES IN THE WORKPLACE}

${ }^{1} \mathrm{~N}$ Loio-Marques*, ${ }^{2} \mathrm{M}$ Felgueiras, ${ }^{3} \mathrm{C}$ Turbau, ${ }^{4} \mathrm{R}$ Valls-Llobet. ${ }^{1}$ Occupational Medicine Resident, Centro Hospitalar Porto, Porto, Portugal; ${ }^{2}$ Clinical Pathology Resident, Centro Hospitalar Cova Beira, Portugal; ${ }^{3}$ Biologist, Centre d'Atenció Programes Sanitaris (CAPS), Barcelona, España; ${ }^{4}$ Endocrinologist, CAPS, Barcelona, España

\subsection{6/oemed-2018-ICOHabstracts. 1120}

Introduction Over the last decades a relationship has been established between the use of organophosphates (OP) and the development of systemic symptomatology, possibly resulting in the diagnostic of Multiple Chemical Sensitivity Syndrome (MCSS), Chronic Fatigue Syndrome (CFS), Mitochondrial Dysfunction Syndrome (MDS), Chronic OP Induced Neuropsychological Syndrome (COPINS), among others. MD, evidenced by the change in Lactate/Pyruvate ratio, seems to be the set-point of evolution of these syndromes. For MQSS in particular, the diagnosis is done using the internationally acknowledged questionnaire 'Quick Environmental Exposure and Sensitivity Inventory' (QEESI). The main objective of this study was to identify the different diagnosis presented by women chronically exposed to OPs in their workplace.

Methods Continuous observational study (1994-2014) about diagnosis and employment condition of women exposed to OPs.

Results All women showed signs of MCSS, CFS and COPINS. Among the signs and symptoms associated with these syndromes, digestive changes $46 \%$ and food intolerances $12 \%$ were the most observed. Also, $62 \%$ of the women showed a sign described as typical from intoxication by OPs: black tongue. The results also show that all women had mitochondrial dysfunction.

Conclusion Exposure to OPs seems to contribute towards MCSS, CFS, MDS and other systemic symptoms of a chronic and disabling character, with the possibility of having associated mitochondrial dysfunction as the set-point.

\section{NAFLD AND OM PRACTICE}

MD Renales*. Consultant, Minute Healthcare, Quezon City, Philippines

\subsection{6/oemed-2018-ICOHabstracts.1121}

Introduction Non-alcoholic fatty liver disease is an inflammation of the liver caused by an accumulation of fat deposits in the liver tissue. The common underlying problem is obesity, diabetes, or both in metabolic syndrome. NAFLD is manifested by an increase in alanine aminotransferase in liver function tests. Progressive disease results in cirrhosis if the cause remains untreated. Currently, NAFLD is an increasing problem in Asia as well as in the Philippines.

Methods This is a review of literature on the emerging problem of NAFLD. Interventions focus on the individual and enterprise management of NAFLD.

Result Prevalence of NAFLD is $12.2 \%$ in a hospital-based Philippine study. The characteristic features included female, sex, obesity, elevated liver enzymes and diabetes. Another study compared ASH, NASH, and TASH and how disease progression is a result of both direct effects on the liver as well as indirect alterations in other organs and tissues such as intestine, adipose tissue, and immune system. In another study, the increasing incidence of NAFLD in Western countries is strictly linked to lifestyle habits. The prevalence of NAFLD in European and Japanese population-based studies is estimated to be $14 \%$ to $21 \%$. One study presents lower prevalence that is variable between $15 \%$ to $20 \%$ in Asian countries than the Western countries.

Discussion Using the above results of the literature review, the paper discusses occupational management of individuals with NAFLD as well as the enterprise as a whole.

Conclusion The occupational physician coordinates with the external physician for care of diagnosed workers with NAFLD, work accommodation, and fitness to return to work. Workplace management approaches discuss interventions to reduce if not prevent NAFLD due to workplace toxic and lifestyle agents. 